\title{
Anterior chamber depth correlated with anthropomorphic measurements: the Beijing Eye Study
}

\begin{abstract}
Background To assess the relationship between peripheral anterior chamber depth and anthropomorphic measurements.

Methods The Beijing Eye Study 2006 included $3251(73.2 \%)$ subjects who returned for a followup examination after a baseline examination including 4335 subjects in 2001. The peripheral anterior chamber depth was estimated by slit lamp-assisted biomicroscopy using van Herick's method. Measurements of the body height and weight and assessment of the anterior chamber as inclusion criterion for this study were available for $3191(\mathbf{9 8 . 2 \%})$ subjects.

Results In a multivariate analysis, a shallow peripheral anterior chamber was significantly associated with higher age $(P<0.001)$, female gender $(P<0.001)$, and shorter height $(P<0.001)$, whereas weight $(P=0.97)$ and body mass index $(P=0.82)$ were not significantly associated.

Conclusions The peripheral anterior chamber depth was inversely correlated with height, ie, a shallow peripheral anterior chamber may be expected with a higher frequency in short subjects than in tall subjects. Height, in addition to higher age and female gender, may be taken as one of the parameters for screening of subjects being at risk for primary angleclosure glaucoma.
\end{abstract}

Eye (2009) 23, 632-634; doi:10.1038/eye.2008.26; published online 29 February 2008

Keywords: anterior chamber depth; Beijing Eye Study; anthropomorphic measurements

\section{Introduction}

Previous studies have shown that a shallow anterior chamber is a risk factor for the development of primary angle-closure glaucoma. ${ }^{1}$ Other investigations have revealed that primary angle-closure glaucoma is markedly more common in east Asian countries than in western societies, so that screening examinations for the detection of subjects at risk to develop angle-closure glaucoma are recommendable for the east Asian countries., ${ }^{2,3}$ As it is useful for a screening process to have a parameter, which helps to differentiate subjects at risk for the disease from those are not, and because the anterior chamber depth is not easily assessed unless a skilled examiner and a slit lamp are available, it was the purpose of the present investigation in China to examine whether a shallow anterior chamber, as marker of a small eye, may be associated with a short body stature, which could be easily assessed.

\section{Methods}

The Beijing Eye Study is a population-based study, which was performed in 2001 and included 4439 subjects out of 5324 subjects invited to participate with an age of $40+$ years (response rate: $83.4 \%$ ). The study has been described in detail previously. ${ }^{4}$ The Medical Ethics Committee of the Beijing Tongren Hospital had approved the study protocol and all subjects had given informed consent, according to the Declaration of Helsinki. In 2006, all study subjects were invited to be re-examined. Out of the 4439 subjects, 3251 $(73.2 \%)$ returned for the re-examination, while $144(3.2 \%)$ had died and $1044(23.5 \%)$ refused to participate or had moved away. The examinations included a slit-lamp examination carried out by an ophthalmologist. The peripheral anterior chamber depth was assessed using van Herick's method. ${ }^{5}$ Using the slit 
lamp, the peripheral depth of the anterior chamber close at the limbus was estimated by directing the beam of the slit lamp perpendicularly to the corneal surface, and the depth of the anterior chamber was assessed using the corneal thickness as relative size unit. A grading scale was used with grade ' 4 ' for a widely open anterior chamber angle (chamber depth $>\frac{1}{2}$ of the corneal thickness), and grade ' 1 ' for a 'very narrow anterior chamber angle' (chamber depth $<\frac{1}{4}$ of the corneal thickness). Measurements of height and weight and assessment of the anterior chamber as inclusion criterion for this study were available for 3191 (98.2\%) subjects.

Statistical analysis was performed by using a commercially available statistical software package (SPSS for Windows, version 15.0, SPSS, Chicago, IL. USA). For the statistical analysis, the actual height measurements and not percentiles were taken. Only one randomly selected eye per subject was taken for the statistical analysis.

\section{Results}

The distribution of anterior chamber angle according to body height and gender was showed in Table 1. In univariate analysis, peripheral anterior chamber depth as assessed by van Herick's method was significantly associated with younger age $(P<0.001$; correlation coefficient $r=0.16$; slope of the regression line: -0.015 ; 95\% confidence interval of the slope $(\mathrm{CI}):-0.017$, $-0.013)$, male gender $(P<0.001 ; 95 \% \mathrm{CI}$ of the difference; $0.18,0.27)$, height $(P<0.001 ; r=0.16$; slope: $0.018 ; 95 \% \mathrm{CI}$ : $0.014,0.022)$, and weight $(P<0.001 ; r=0.10$; slope: 0.008 ; $95 \%$ CI: $0.005,0.011)$. In a multivariate analysis, with peripheral anterior chamber depth as dependent variable and age, height, weight, and gender as independent variables, peripheral anterior chamber depth was still significantly associated with younger age $(P<0.001$;

Table 1 Peripheral anterior chamber depth measurements as assessed by van Herick's method in the Beijing Eye Study 2006, in categories of age and gender

\begin{tabular}{llrc}
\hline Body height $(\mathrm{cm})$ & Gender & $N$ & $\begin{array}{c}\text { Anterior chamber Angle } \\
\text { (van Hericks method) }\end{array}$ \\
\hline$<150$ & Male & 5 & 4.0 \\
$150-159$ & Female & 235 & $3.10 \pm 1.23$ \\
& Male & 150 & $3.64 \pm 0.87$ \\
$160-169$ & Female & 1078 & $3.47 \pm 1.03$ \\
& Male & 760 & $3.74 \pm 0.74$ \\
$170-179$ & Female & 468 & $3.61 \pm 0.89$ \\
$180+$ & Male & 452 & $3.80 \pm 0.67$ \\
& Female & 18 & $3.89 \pm 0.47$ \\
& Male & 24 & $3.88 \pm 0.61$ \\
\hline
\end{tabular}

coefficient: $-0.015,95 \%$ CI: $-0.018,-0.012)$, male gender $(P<0.001$; coefficient: $-0.16 ; 95 \% \mathrm{CI}:-0.025,-0.007)$, and taller height $(P<0.001$; coefficient: 0.010 ; 95\% CI: 0.004 , $0.015)$, while weight was no longer associated with the anterior chamber depth $(P=0.97)$. Consequently, the body mass index was not significantly associated with anterior chamber depth in univariate analysis $(P=0.82)$.

\section{Discussion}

The results suggest that anterior chamber depth was inversely correlated with height, ie, a shallow anterior chamber may be expected with a slightly higher frequency in short subjects than in tall subjects. It confirms a recent report from the Beijing Eye Study, in which subjects with angle-closure glaucoma were significantly shorter in stature than the subjects without angle-closure glaucoma. ${ }^{6}$ This study confirms a previous investigation on Singaporeans, in which, as in this study, an inverse correlation between anterior chamber depth and height was found. ${ }^{7}$ It is also in agreement with the recent Meiktila Eye Study from Myanmar and the Reykjavik Eye Study from Iceland in both of which height was associated with the ocular dimensions. ${ }^{8,9}$ Height, in addition to higher age and female gender, may therefore be taken as one of the parameters for screening of subjects being at risk for angle-closure glaucoma. It has to be taken into account, however, that only this study identified an associated factor (short height) for a risk factor of angle-closure glaucoma. Future studies may address the direct association between height $v$ s anterior chamber angle width and may further examine whether the inclusion of height into the list of screening parameters indeed improves the performance of screening investigations for angle-closure glaucoma.

\section{Acknowledgements}

This study was supported by the Beijing Nature Science Foundation.

\section{References}

1 Congdon NG, Youlin O, Quigley HA, Hung PT, Wang TH, Ho TC et al. Biometry and primary angle-closure glaucoma among Chinese, white, and black populations. Ophthalmology 1997; 104: 1489-1495.

2 Foster PJ, Oen FT, Machin D, Ng TP, Devereux JG, Johnson GJ et al. The prevalence of glaucoma in Chinese residents of Singapore. Arch Ophthalmol 2000; 118: 1105-1111.

3 Jacob A, Thomas R, Koshi SP, Braganza A, Muliyil J. Prevalence of primary glaucoma in an urban south Indian population. Indian J Ophthalmol 1998; 46: 81-86. 
4 Xu L, Wang Y, Wang S, Wang Y, Jonas JB. High myopia and glaucoma susceptibility: the Beijing Eye Study. Ophthalmology 2007; 114: 216-220.

5 Van Herick W, Shafer RN, Schwartz A. Estimation of width of angle of anterior chamber: incidence and significance of the narrow angle. Am J Ophthalmol 1969; 68: 626-629.

$6 \mathrm{Xu} \mathrm{L,} \mathrm{Li} \mathrm{J,} \mathrm{Wang} \mathrm{Y,} \mathrm{Jonas} \mathrm{JB.} \mathrm{Anthropomorphic} \mathrm{differences}$ between angle-closure versus open-angle glaucoma: the Beijing Eye Study.

7 Wong TY, Foster PJ, Johnson GJ, Klein BE, Seah SK. The relationship between ocular dimensions and refraction with adult stature: the Tanjong Pagar Survey. Invest Ophthalmol Vis Sci 2001; 42: 1237-1242.

8 Wu HM, Gupta A, Newland HS, Selva D, Aung T, Casson RJ. Association between stature, ocular biometry and refraction in an adult population in rural Myanmar: the Meiktila eye study. Clin Experiment Ophthalmol 2007; 35: 834-839.

9 Eysteinsson T, Jonasson F, Arnarsson A, Sasaki H, Sasaki K. Relationships between ocular dimensions and adult stature among participants in the Reykjavik Eye Study. Acta Ophthalmol Scand 2005; 83: 734-738. 